\title{
Experimental evidence of an environmental attitude-behavior gap in high-cost situations
}

\author{
Mike Farjam, Olexandr Nikolaychuk, Giangiacomo Bravo*
}

\begin{abstract}
So far, there has been mixed evidence in the literature regarding the relation between environmental attitudes and actual "green" actions, something known as attitude-behavior gap. This raises the question of when attitudes can actually work as a lever to promote environmental objectives, such as climate change mitigation, and, conversely, when other factors would be more effective. We tested the effect of environmental attitudes on behavior in an online experiment with real money at stake and real-world consequences. We found that environmental attitudes affected behavior in low-cost situations while increasing contribution costs generally reduced their effect. This finding is consistent with the low-cost hypothesis of environmental behavior and has important consequences for the design of more effective climate policies in a democratic context.
\end{abstract}

Keywords: Climate-change mitigation, Low-cost hypothesis, Online experiment, Collectiverisk social dilemma

\section{Introduction}

A established research finding is that environmental attitudes of people only loosely translate into actions able to effectively reduce their environmental impact, something known as an attitudebehavior gap [1-4]. This is especially relevant in the context of climate change mitigation, where unsustainable behaviors are maintained in the face of increasing evidence for the necessity of a rapid change [5,6], and even policy makers seem unwilling to propose the most effective actions to the public, possibly fearing their unpopularity [7]. On the other hand, dissemination of correct information and environmental education are considered key to promote sustainability [8-10]. This raises the question of when attitudes can actually work as a lever to promote environmental objectives and, conversely, when other factors could be more effective. To answer it, we tested the effect of environmental attitudes in an online experiment with real money at stake and real-world climate mitigation consequences. We found that environmental attitudes affected behavior in lowcost situations while increasing contribution costs generally reduced their effect. This finding is consistent with the low-cost hypothesis of environmental behavior [11,12] and has important consequences for the design of more effective climate policies in a democratic context $[5,13]$.

According to the low-cost hypothesis of environmental behavior, environmental attitudes only promote "green" actions when the related behavioral costs are low [11,14]. In practice, this means that concerned people are likely to take actions that are easy to perform or do not require excessive investments, such as switching off the lights or buying organic food. However, the effect of environmental attitudes is reduced when the actions imply considerable monetary or behavioral costs-e.g., not driving to work or reducing air travel- despite significantly higher benefits for the

\footnotetext{
*Department of Social Studies and Center for Data Intensive Sciences and Applications, Linnaeus University, Växjö, Sweden

${ }^{\dagger}$ Faculty of Economics and Business Administration, Friedrich Schiller University, Jena, Germany
} 
environment. Diekmann and Preisendörfer suggested that concerned people use low-cost actions to reduce the cognitive dissonance between their concern for the environment and rational realisation of the environmental impact of their actions, while avoiding costly actions despite their higher effectiveness [14].

Much of past research on the low-cost hypothesis was survey-based and led to contradictory findings $[15,16]$. The few existing natural experiments and intervention studies, often focusing on recycling behavior, provided mixed evidence, too, suggesting that environmental attitudes may only predict behavior in select contexts $[17,18]$ or under specific conditions [19]. Overall, the diversity of factors affecting behavior makes a clean test of the hypothesis in natural settings difficult if not impossible.

\section{Methods}

In order to make sense of these contradictory findings in a more controlled setting, we conducted an experiment with real-world incentives on a sample of adult US residents, which allowed for a proper test of the relationship between attitudes and costs on one side and actual behavior on the other. The experiment was based on an established protocol-the collective-risk social dilemma (CRSD) [20] - that provided several advantages. First, the CRSD has been tested in a number of studies which, among other things, expanded the original design to include such factors as inequality [21], ambiguous thresholds [22] and residual risk [23]. Second, not only does the protocol have a clear frame of climate change mitigation but the participants' actions have an actual positive impact on the environment (in our study, the purchasing of carbon offset certificates), all while maintaining a rigorous experimental structure. This enabled the participants with actual environmental concerns to make incentivized decisions with real environmental consequences in a controlled setting.

In our CRSD game, six players made individual decisions over the course of 10 rounds on whether or not to contribute to what was called the climate account $(C)$. Each player had an initial endowment of 40 experimental currency units (ECU) and in each round, could contribute 0,2 , or 4 ECU to the climate account. The contributions were public, not refundable, and whether or not a group threshold of $C=120 \mathrm{ECU}$ was reached by the end of the game affected how the payoffs were determined. In case of success (i.e., $C \geq 120 \mathrm{ECU}$ ), the players received what remained of their endowments. In case of failure (i.e., $C<120 \mathrm{ECU}$ ), they faced a common risk of losing what remained of their endowments with a predetermined and known probability $p$. As an emulation of the real world scenario, where it is estimated that $\mathrm{CO}_{2}$ must be reduced by about $45 \%$ from its 2010 level by the year 2030 in order to prevent the worst consequences of climate change [24], the participants were told that an average individual contribution of 2 ECU per round was needed to reach the group threshold (i.e., a total contribution of half of the individual endowment by the end of the game).

Following the original parametrisation of the CRSD game [20], we considered three levels of risk: $p \in\{0.1,0.5,0.9\}$. In addition, we varied the conversion rate between ECU and USD across the treatments: 10:1 and 1:1 in the low-cost and high-cost conditions, respectively. The participants also filled out a post-experiment questionnaire with items assessing their environmental concern and positioning on the left/right-wing political scale (see Appendix). The contributions to the climate account had real-world consequences in that they were used to finance carbon offset initiatives. In total, an equivalent of 4800 USD was contributed to offset $14,500 \mathrm{t}$ of $\mathrm{CO}_{2}$ via the United Nations platform for voluntary cancellation of certified emission reductions.

Within the scope of our experimental design, the low-cost hypothesis predicted that, in the low-cost conditions, the participants with a high level of environmental concern should contribute more than those with a low level of environmental concern. In the high-cost conditions, due to the 
tenfold increase of the contribution cost, one's environmental concern should matter considerably less, if at all. More specifically, the low-cost hypothesis led to the following predictions in our setup (See Appendix): (i) a positive main effect of environmental concern on the contribution to the climate account; (ii) a negative effect on the contribution of the interaction between environmental concern and costs, potentially negating the first effect completely in the high-cost conditions.

\section{Results}

A total of 714 US residents, recruited via the crowd-sourcing platform Mechanical Turk (MTurk), participated in the experiment. From the analysis, we excluded all groups with at least one participant not finishing the game or not filling out the questionnaire (see Appendix), which led to a total of 660 players in 110 groups. Figure 1 shows an overview of the general time trends of the contributions over the 10 rounds of the CRSD game. The vertical axis measures the distance from the reference, defined as the difference between one's total contribution up to and including the current round and the reference scenario, where each participant contributes 2 ECU per round (see Appendix). The contributions were generally higher and the differences between the various levels of risk were more pronounced in the high-cost conditions.

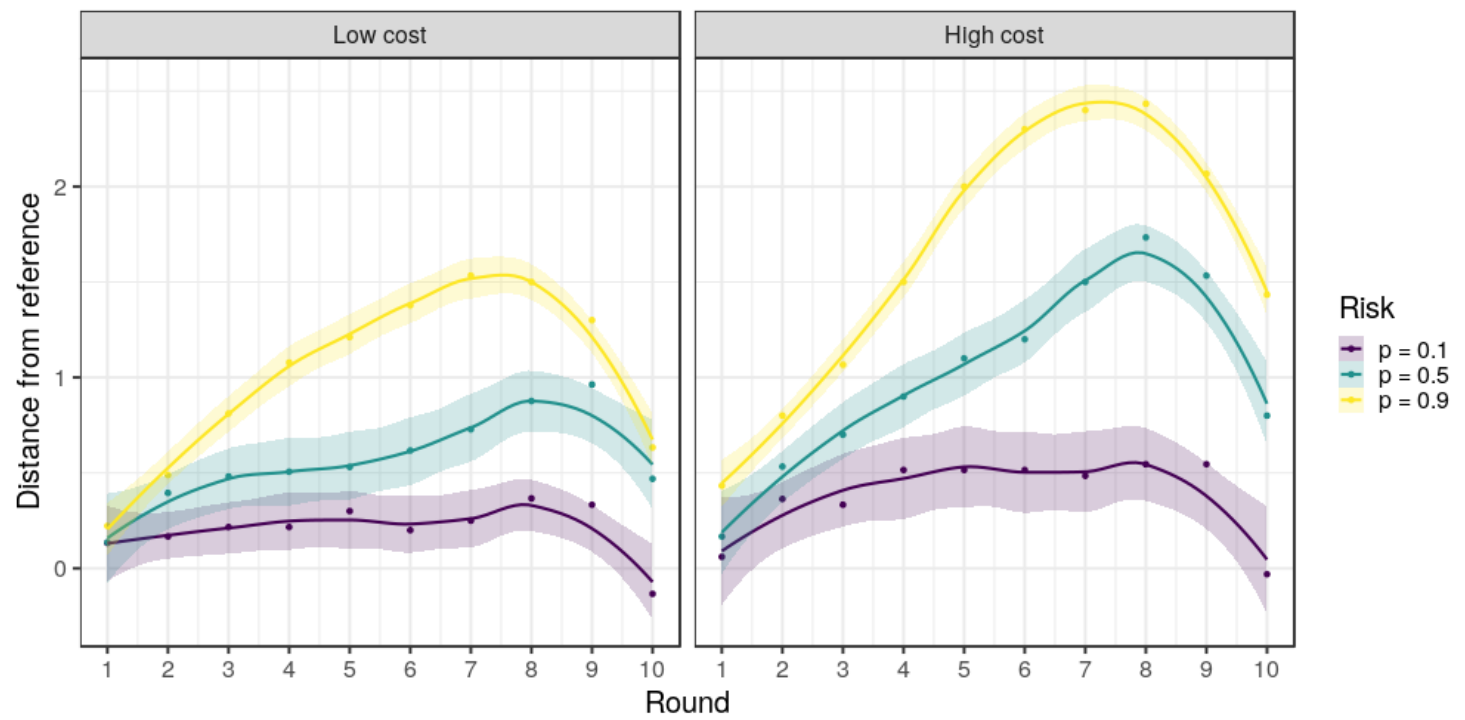

Figure 1: Average cumulative distance (in experimental currency units) from the reference individual contribution of 2 per treatment. The splines represent smoothed trends with $95 \%$-confidence bands.

Figure 2 shows the average contributions in the low- and high-cost conditions, separately for the participants with low and high levels of environmental concern (see Appendix). In line with the low-cost hypothesis, the participants with a high level of environmental concern contributed more to the climate account in the low-cost conditions, while there was no apparent difference in the high-cost conditions.

In order to formally test the predictions of the low-cost hypothesis, we used a mixed-effects ordered logit regression model, with the individual contribution to the climate account in each round as the dependent variable (see Appendix, Eq. 2). To account for the common (non-linear) time trend, the model included fixed effects for round and round squared. Since the low-cost hypothesis predicted a negative interaction effect between environmental concern and cost of supporting climate change mitigation activities, the model included fixed effects for both, as well as for their 


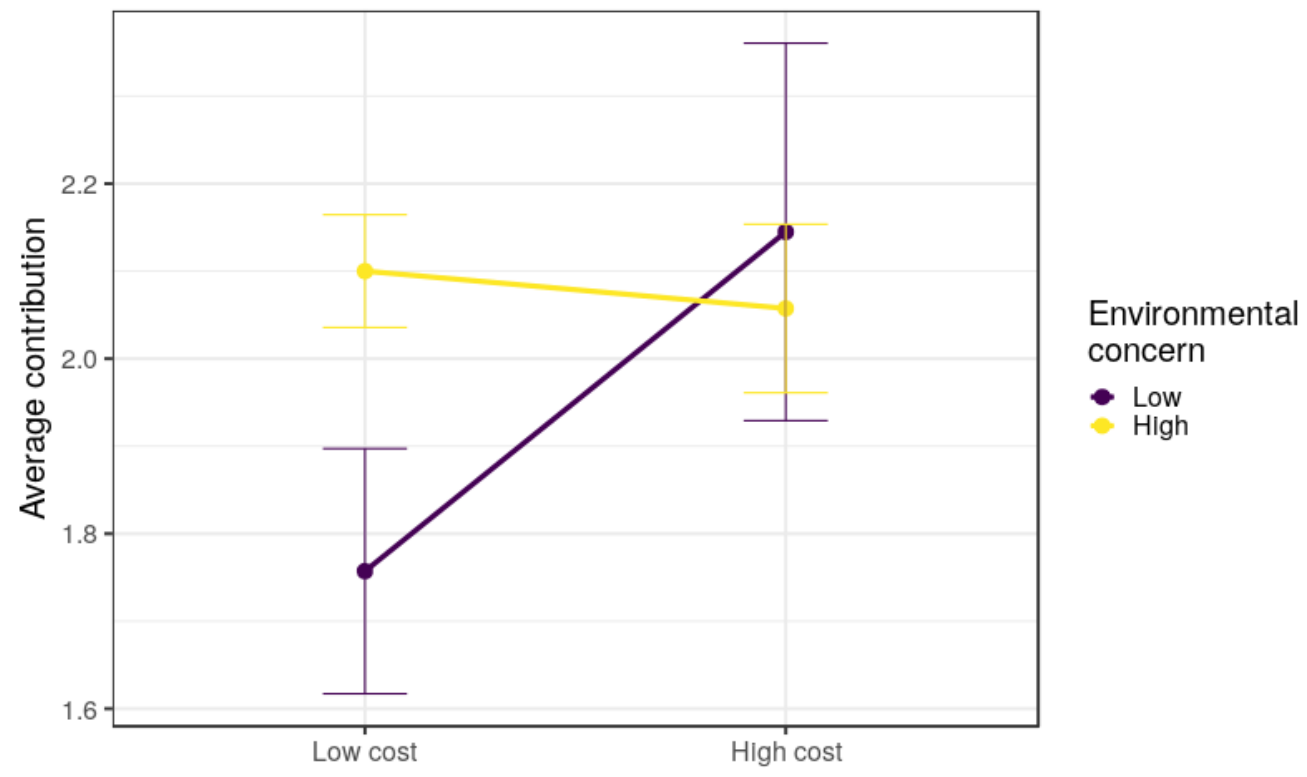

Figure 2: Average individual contribution (in experimental currency units), separately for the participants with low and high levels of environmental concern. The whiskers represent the standard errors.

interaction. As the last set of fixed effects, we added binary variables capturing the various levels of risk faced by the participants. Finally, to account for the possible correlation structure within the data, the model included random effects at the group and participant level.

We adopted the Bayesian framework with vague priors (see Appendix) to fit the data to the model, and the results are presented in Table 1 (Model 1). As indicated by the signs of the coefficient estimates and the associated Bayes factors, we found very strong evidence [25] in favour of a positive effect of environmental attitude on contribution behavior as well as strong evidence in favour of a negative interaction effect between environmental attitude and costs, which supports the low-cost hypothesis. In addition, we found positive evidence in favour of a positive effect of risk level, which is in line with most replications of the CRSD game [20-23].

\begin{tabular}{|c|c|c|c|c|c|c|}
\hline & \multicolumn{3}{|c|}{ Model 1} & \multicolumn{3}{|c|}{ Model 2} \\
\hline & Estimate & {$\left[\mathrm{CI}_{95}\right]$} & $\begin{array}{l}\text { Bayes } \\
\text { factor }\end{array}$ & Estimate & {$\left[\mathrm{CI}_{95}\right]$} & $\begin{array}{l}\text { Bayes } \\
\text { factor }\end{array}$ \\
\hline $\mathrm{p}=0.5$ & 0.15 & {$[-0.14 ; 0.47]$} & $1: 5$ & 0.16 & {$[-0.15 ; 0.48]$} & $1: 5$ \\
\hline $\mathrm{p}=0.9$ & 0.18 & {$[-0.11 ; 0.48]$} & $1: 7$ & 0.19 & {$[-0.11 ; 0.52]$} & $1: 8$ \\
\hline high-cost & 0.03 & {$[-0.23 ; 0.31]$} & $1: 1$ & 0.05 & {$[-0.22 ; 0.33]$} & $1: 2$ \\
\hline env. & 0.38 & {$[0.24 ; 0.53]$} & $1: 10000$ & & & \\
\hline high-cost $\times$ env & -0.28 & {$[-0.54 ;-0.01]$} & $47: 1$ & & & \\
\hline LWE & & & & 0.34 & {$[0.20 ; 0.49]$} & $1: 10000$ \\
\hline high-cost $\times$ LWE & & & & -0.42 & {$[-0.69 ;-0.13]$} & 624:1 \\
\hline round & 0.24 & {$[0.16 ; 0.32]$} & $1: 10000$ & 0.24 & {$[0.16 ; 0.32]$} & $1: 10000$ \\
\hline round $^{2}$ & -0.03 & {$[-0.04 ;-0.03]$} & 10000:1 & -0.03 & {$[-0.04 ;-0.03]$} & 10000:1 \\
\hline
\end{tabular}

Table 1: Coefficient estimates for the mixed-effects ordered logit regression model of the individual contribution to the climate account. The Bayes factors correspond to the posterior probabilities of the estimates being larger than zero. The coefficients for env. and $L W E$ have been standardized. 
In many countries around the world, and especially so in the US, political affiliations and environmental attitudes tend to correlate, resulting in a growing polarisation on such topics as climate change and environmental protection [26-28]. This was also the case in our sample, where the environmental attitude of the participants was correlated with their self-assessment on the political left/right-wing scale (Spearman's $\rho=-0.44$ ). Since it is quite plausible that the same latent factor underlies the two measures, we aggregated them into one index, dubbed left-wing environmentalism (LWE, see Appendix). By doing so, we hoped to better capture the signal underlying the two individual measures through a reduction of the measurement noise. Model 2 in Table 1 presents the coefficient estimates when using this aggregated index. They are qualitatively similar to the ones found by the first model but provide even stronger evidence as far as the interaction effect.

\section{Discussion}

To sum up, our experiment showed that the effect of environmental attitude on contribution behavior, while positive in principle, depends on the cost of such behavior, which is in line with the prediction of the low-cost hypothesis of environmental behavior. In contrast to earlier works presenting mixed evidence on the issue, by conducting a controlled experiment with monetary incentives and real-world consequences we were able to account for potential confounds and investigate the causal link between costs and contributions to climate change mitigation. An additional degree of realism was warranted by the fact that the study was conducted on a sample of adult US residents. As it stands, perhaps the only major limitations were the duration of the experiment and the relative abstractness of contribution behavior since the actual climate 'game' is being played on a considerably larger time scale and presupposes real physical (inter)actions. Nevertheless, the practical relevance of the insights provided by the CRSD protocol has been repeatedly shown both in the social and environmental research literature [20-23], which also applies to our study.

The next logical step would be to investigate if the low-cost hypothesis could be extended to other domains. Indeed, it seems quite plausible that the same mechanism could be at work in different contexts. For instance, many Germans supported, among other things financially, the inflow of migrants during the 'refugee crisis' of 2015. Their willingness to pay for such programs, however, appears to be negatively related to the number of immigrants in their close proximity, possibly due to an increase in the associated costs of the local welfare systems [29]. This is just one example of a wide variety of phenomena to explore in future experiments, so that the low-cost hypothesis could in principle be generalized beyond its original domain.

Coming back to the main research question, our findings put forward the idea that reinforcing environmental concern through communication and education is going to have a meaningful effect in basic, low-cost situations, whereas stronger incentives (e.g., carbon taxes) and/or structural changes (e.g., investment into green infrastructure) are required when the scope and the associated cost of the necessary changes increase. That said, raising concern may still have indirect positive effects, for instance by increasing support for more ambitious environmental policies [14]. More generally, instead of proclaiming the desired effects of tentative policies or deriving rational predictions under simplistic modelling assumptions, we argue that it is important to test prospect changes using rigorous experimental methods [30]. This way, the design and acceptance of climate and energy policies could be greatly enhanced, while mistakes and counter-reactions potentially undermining their effects if not completely backfiring — as illustrated by the 2018 events in France where public opposition to a new carbon tax on transportation fuels forced the government to rethink its strategy [31]— could be prevented [30,32].

Acknowledgements. The research presented in this article was supported by the Linnaeus University Center for Data Intensive Sciences and Applications, DISA (http://lnu.se/disa). This paper 
has profited from comments by Marc Keuschnigg.

Author contributions. M.F., O.N. and G.B. planned the research, discussed the results and wrote the paper. M.F. and O.N. coded and conducted the experiment and analyzed the data.

\section{A Additional Materials and Methods}

\section{A.1 Experiment implementation}

The participants were recruited via Amazon Mechanical Turk (MTurk) and the experiment was programmed using oTree [33]. Only adult US residents were allowed to participate. The average payment was 3.4 USD in the low-cost conditions and 21.5 USD in the high-cost conditions (including a "show up" fee of 1.3 USD) for an average duration of 15 minutes. As a means of control as well as to rule out the potential use of software bots, the participants were required to provide correct answers to at least 3 out of 7 comprehension questions.

The participants received on-screen instructions similar to those in [23], which included a brief explanation of the problem of global warming and use of the funds contributed to the climate account. If qualified, they would play 10 periods of the common-risk social dilemma game and finally, would be asked to fill out a short, non-incentivized questionnaire eliciting their political orientation and environmental concern using questions derived from the World Value Survey [34].

Altogether, 119 groups of participants were recruited for the experiment. Treatment allocation was randomized, and the game was played only once. All player interaction was anonymized. Out of the 199 groups of participants, 9 had at least one member who become inactive at some point during the session and, as such, were excluded from the analysis. In the end, the statistical analysis encompassed 660 participants, or 110 groups worth of data.

\section{A.2 Note on cost manipulation}

As a side effect of relying on the CRSD protocol, the individual endowment, not just the cost of contribution, had to be scaled up in the high-cost conditions (both depend on the conversion rate between ECU and USD). While this could be considered as a technical limitation, we believe that given the context of the decision situation, the participant motivations were not limited to the pecuniary incentives provided by the endowment, and that the benefits of staying within an established paradigm make it worthwhile in the end. To operationalize the predictions of the lowcost hypothesis within our setup, we included the main effect of the cost manipulation as a binary variable, as well as its interaction with environmental concern (Eq. 2).

\section{A.3 Distance from reference}

To account for the fact that in the collective risk social dilemma game the participants have to make decisions repeatedly before the final outcome is realized, instead of taking the contributions at their face value we followed previous research [23] in considering the cumulative distance from the reference contribution as the dependent variable in Figure 1.

The distance from the reference was computed for each participant and round as follows:

$$
\Delta_{i}^{t}=\sum_{\tau=1}^{t} C_{i}^{\tau}-2 t,
$$

where $i$ and $t$ respectively index the participant and round, and $C_{i}^{\tau} \in\{0,2,4\}$ denotes the contribution of participant $i$ in round $\tau, \tau \in\{1,2, \ldots, t-1, t\}$. The number 2 represents the fair 
reference contribution needed to reach the group-level threshold of 120 by the end of the game. One key advantage of this metric is that it makes it clear if a given participant is "on track" during the course of the game.

\section{A.4 Environmental attitude index}

Environmental attitude, or concern, was elicited via the following question: "Generally speaking, how concerned are you about environmental issues?" The answers were measured using a 0-10 scale where zero corresponded to "not at all concerned" and ten corresponded to "very concerned". There was no default or otherwise focal answer indicated.

Figure 2 was constructed by splitting the participants into two groups as far as the index imputed from their answer. They were assigned to have a high level of environmental concern for values larger than five and a low level of environmental concern otherwise.

\section{A.5 Statistical analysis}

To test the statistical significance of the findings, we estimated a mixed-effects ordered logit model with the latent dependent variable specified as follows:

$$
\begin{aligned}
y_{g, i, t}^{*}= & \beta_{1} \cdot \operatorname{round}_{t}+\beta_{2} \cdot \operatorname{round}_{t}^{2}+\beta_{3} \cdot P 5_{g}+\beta_{4} \cdot P 9_{g}+\beta_{5} \cdot H C_{g}+\beta_{6} \cdot E N V_{g, i}+ \\
& +\beta_{7} \cdot H C \times E N V_{g, i}+v_{g}+v_{g, i}^{\prime}+\varepsilon_{g, i, t},
\end{aligned}
$$

where $g, i$ and $t$ respectively index the group, participant within the group and round; $P 5_{g}$ and $P 9_{g}$ equal one if the group is assigned to the treatment with a moderate $(p=0.5)$ or high $(p=0.9)$ level of risk and zero otherwise ( $p=0.1$ ); $H C_{g}$ equals one if the group is assigned to the high-cost treatment $(1 \mathrm{ECU}=1 \mathrm{USD})$ and zero otherwise $(10 \mathrm{ECU}=1 \mathrm{USD}) ; E N V_{g, i}$ captures the individual environmental concern (Model 1) or the left-wing environmentalism (Model 2). Finally, $v_{g}$ and $v_{g, i}^{\prime}$ represent the random effect at the group and participant level, respectively.

Both the individual environmental concern and political orientation were measured using a 0-10 scale, where zero corresponded to "low concern" and "right-wing", respectively. The leftwing environmentalism was constructed as the difference between the two such that zero would correspond to "low concern". The resulting indices were subsequently standardized.

To fit the data to the model, we adopted the Bayesian framework with vague priors and estimated the posterior distributions of the coefficients using the brms package [35] (version 2.7.0) for the $R$ software environment [36] (version 3.4.4). The results were based on sampling from two independent chains with $5000+5000$ samples each.

\section{A.6 Data availability}

All data and scripts needed to reproduce the analysis can be downloaded from https://dataverse. harvard.edu/privateurl.xhtml?token=6d7b7275-efac-414e-bd14-7a33f4e941a5

\section{References}

[1] Michal J. Carrington, Benjamin A. Neville, and Gregory J. Whitwell. Lost in translation: Exploring the ethical consumer intention-behavior gap. Journal of Business Research, 67(1):2759-2767, jan 2014. 
[2] Anja Kollmuss and Julian Agyeman. Mind the gap: Why do people act environmentally and what are the barriers to pro-environmental behavior? Environmental Education Research, 8(3):239-260, aug 2002.

[3] Louise M. Hassan, Edward Shiu, and Deirdre Shaw. Who says there is an intention-behaviour gap? assessing the empirical evidence of an intention-behaviour gap in ethical consumption. Journal of Business Ethics, 136(2):219-236, nov 2014.

[4] Ken Peattie. Green consumption: Behavior and norms. Annual Review of Environment and Resources, 35(1):195-228, nov 2010.

[5] Irene Lorenzoni, Sophie Nicholson-Cole, and Lorraine Whitmarsh. Barriers perceived to engaging with climate change among the UK public and their policy implications. Global Environmental Change, 17(3-4):445-459, aug 2007.

[6] Lorraine Whitmarsh. Behavioural responses to climate change: Asymmetry of intentions and impacts. Journal of Environmental Psychology, 29(1):13-23, mar 2009.

[7] Seth Wynes and Kimberly A Nicholas. The climate mitigation gap: education and government recommendations miss the most effective individual actions. Environmental Research Letters, 12(7):074024, jul 2017.

[8] Matthew C. Nisbet. Communicating climate change: Why frames matter for public engagement. Environment: Science and Policy for Sustainable Development, 51(2):12-23, mar 2009.

[9] A. E. J. Wals, M. Brody, J. Dillon, and R. B. Stevenson. Convergence between science and environmental education. Science, 344(6184):583-584, may 2014.

[10] Anne Kristin Liefländer and Franz Xaver Bogner. The effects of children's age and sex on acquiring pro-environmental attitudes through environmental education. The Journal of Environmental Education, 45(2):105-117, feb 2014.

[11] Andreas Diekmann and Peter Preisendörfer. Green and greenback: The behavioral effects of environmental attitudes in low-cost and high-cost situations. Rationality and Society, 15(4):441-472, 2003.

[12] Paul C. Stern. Toward a coherent theory of environmentally significant behavior. Journal of Social Issues, 56(3):407-424, 2000.

[13] Nico Stehr. Climate policy: Democracy is not an inconvenience. Nature, 525:449-450, 2015.

[14] Andreas Diekmann and Peter Preisendörfer. Environmental behavior: Discrepancies between aspirations and reality. Rationality and Society, 10(1):79-102, 1998.

[15] Florian G. Kaiser and P. Wesley Schultz. The attitude-behavior relationship: A test of three models of the moderating role of behavioral difficulty1. Journal of Applied Social Psychology, 39(1):186-207, jan 2009.

[16] Peter Kriwy and Rebecca-Ariane Mecking. Health and environmental consciousness, costs of behaviour and the purchase of organic food. International Journal of Consumer Studies, 36(1):30-37, may 2011. 
[17] Henning Best and Thorsten Kneip. The impact of attitudes and behavioral costs on environmental behavior: A natural experiment on household waste recycling. Social Science Research, 40(3):917-930, may 2011.

[18] Pietro Lanzini and John Thøgersen. Behavioural spillover in the environmental domain: An intervention study. Journal of Environmental Psychology, 40:381-390, dec 2014.

[19] Marc Keuschnigg and Fabian Kratz. Thou shalt recycle: How social norms of environmental protection narrow the scope of the low-cost hypothesis. Environment and Behavior, 50(10):1059-1091, oct 2017.

[20] Manfred Milinski, Ralf D. Sommerfeld, Hans-Jurgen Krambeck, Floyd A. Reed, and Jochem Marotzke. The collective-risk social dilemma and the prevention of simulated dangerous climate change. Proceedings of the National Academy of Sciences, 105(7):2291-2294, 2008.

[21] Alessandro Tavoni, Astrid Dannenberg, Giorgos Kallis, and Andreas Löschel. Inequality, communication, and the avoidance of disastrous climate change in a public goods game. Proceedings of the National Academy of Sciences, 108(29):11825-11829, 2011.

[22] Astrid Dannenberg, Andreas Löschel, Gabriele Paolacci, Christiane Reif, and Alessandro Tavoni. On the provision of public goods with probabilistic and ambiguous thresholds. Environmental and Resource Economics, 61(3):365-383, 2015.

[23] Mike Farjam, Olexandr Nikolaychuk, and Giangiacomo Bravo. Does risk communication really decrease cooperation in climate change mitigation? Climatic Change, 149(2):147158, jun 2018.

[24] IPCC. Global warming of $1.5^{\circ} \mathrm{C}$ : An IPCC special report on the impacts of global warming of $1.5^{\circ}$ above pre-industrial levels and related global greenhouse gas emission pathways, in the context of strengthening the global response to the threat of climate change, sustainable development, and efforts to eradicate poverty. Working Group III Contribution to the IPCC Fifth Assessment Report, 2018.

[25] Robert E Kass and Adrian E Raftery. Bayes factors. Journal of the American Statistical Association, 90(430):773-795, 1995.

[26] Aaron M. McCright, Chenyang Xiao, and Riley E. Dunlap. Political polarization on support for government spending on environmental protection in the USA, 1974-2012. Social Science Research, 48:251-260, nov 2014.

[27] Riley E. Dunlap and Aaron M. McCright. A widening gap: Republican and Democratic views on climate change. Environment, 50:26-35, 2008.

[28] Matthew J. Hornsey, Emily A. Harris, and Kelly S. Fielding. Relationships among conspiratorial beliefs, conservatism and climate scepticism across nations. Nature Climate Change, 8(7):614-620, may 2018.

[29] Alexander W. Schmidt-Catran and Dennis C. Spies. Immigration and welfare support in germany. American Sociological Review, 81(2):242-261, mar 2016.

[30] Olivier Oullier. Behavioural insights are vital to policy-making. Nature, 501:463, 2013.

[31] Stefano Carattini, Steffen Kallbekken, and Anton Orlov. How to win public support for a global carbon tax. Nature, 565(7739):289-291, jan 2019. 
[32] Hunt Allcott and Sendhil Mullainathan. Behavior and energy policy. Science, 327:1204$1205,2010$.

[33] Daniel L Chen, Martin Schonger, and Chris Wickens. oTree: An open-source platform for laboratory, online, and field experiments. Journal of Behavioral and Experimental Finance, 9:88-97, 2016.

[34] World Values Survey Association. World values survey wave 6 2010-2014. OFFICIAL AGGREGATE v.20150418. Aggregate File Producer: Asep/JDS, Madrid SPAIN., 2014.

[35] Paul-Christian Bürkner. brms: An R package for Bayesian multilevel models using Stan. Journal of Statistical Software, 80(1):1-28, 2017.

[36] R Core Team. R: A Language and Environment for Statistical Computing. R Foundation for Statistical Computing, Vienna, Austria, 2018. 